\title{
Effective-Component Compatibility of Bufei Yishen Formula $\triangle$ Ameliorated COPD By Improving Airway Epithelial Cell Senescence Via Promoting Mitophagy By Nrf2/PINK1 Pathway
}

\section{Min-yan Li}

Henan Key Laboratory of Chinese Medicine for Respiratory Disease, Henan University of Chinese Medicine, Zhengzhou, Henan

\section{Yan-qin Qin}

Henan Key Laboratory of Chinese Medicine for Respiratory Disease, Henan University of Chinese Medicine, Zhengzhou, Henan

Jian-sheng Li ( $\square$ li_js8@163.com )

Henan Key Laboratory of Chinese Medicine for Respiratory Disease, Henan University of Chinese Medicine, Zhengzhou, Henan

\section{Peng Zhao}

Henan Key Laboratory of Chinese Medicine for Respiratory Disease, Henan University of Chinese Medicine, Zhengzhou, Henan

\section{Yan-ge Tian}

Henan Key Laboratory of Chinese Medicine for Respiratory Disease, Henan University of Chinese Medicine, Zhengzhou, Henan

\section{Kang-chen Li}

Henan Key Laboratory of Chinese Medicine for Respiratory Disease, Henan University of Chinese Medicine, Zhengzhou, Henan

\section{Brian G. Oliver}

School of Life Sciences, Faculty of Science, University of Technology Sydney, Sydney, New South Wales 2007

\section{Xue-fang Liu}

Academy of Chinese Medical Sciences, Henan University of Chinese Medicine

\section{Research Article}

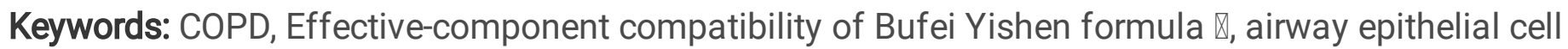
senescence, oxidative stress, mitophagy

Posted Date: January 3rd, 2022 
DOI: https://doi.org/10.21203/rs.3.rs-1198975/v1

License: (c) (1) This work is licensed under a Creative Commons Attribution 4.0 International License. Read Full License 


\section{Abstract}

Background: Effective-component compatibility of Bufei Yishen formula $₫($ (ECC-BYF $\rrbracket)$ shows positive effects on stable chronic obstructive pulmonary disease (COPD).

Purpose: To investigate the mechanisms of ECC-BYF $\triangle$ on COPD rats from the aspect of airway epithelial cell senescence.

Methods: COPD model rats were treated with ECC-BYF $\otimes$ for 8 weeks and the efficacy was evaluated. Cigarette smoke extract (CSE) induced senescence model of airway epithelial cells were treated with ECCBYF $\otimes$, the related enzymes and proteins involved in oxidative stress and mitophagy were detected.

Results: ECC-BYF $\triangle$ markedly rescued pulmonary function and histopathological changes, which might be associated with the amelioration of lung senescence, including reduction of malondialdehyde (MDA) and tumor necrosis factor-a (TNF-a), interleukin (IL)- 6 and matrix metalloproteinase (MMP)-9, increase of the

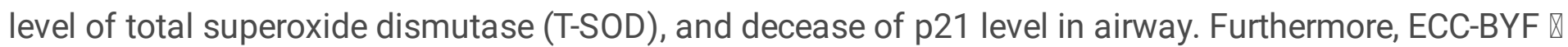
suppressed p16, p21 expressions and senescence-associated $\beta$-galactosidase (SA- $\beta$-Gal) in CSE-induced airway epithelial cells. Moreover, ECC-BYF $\triangle$ upregulated the mitophagy-related proteins, including colocalization of TOM20 and LC3B, PINK1, PARK2, and improved mitochondrial function with upregulating

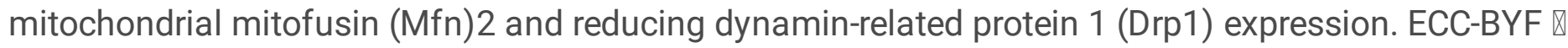
enhanced the activities of T-SOD and GSH-PX by up-regulating Nrf2, thus inhibiting oxidative stress. After intervention with Nrf2 inhibitor, the regulation effects of ECC-BYF $\otimes$ on oxidative stress, mitophagy and senescence in airway epithelial cells were significantly suppressed.

Conclusions: ECC-BYF $\otimes$ exerts beneficial effects on COPD rats by ameliorating airway epithelial cell senescence, which is mediated by inhibiting oxidative stress and subsequently enhancing mitophagy through activation of Nrf2 signaling.

\section{Background}

Chronic obstructive pulmonary disease (COPD) is a heterogeneous disorder involving irreversible airflow restriction and chronic abnormal inflammatory reaction to harmful particles or gases[1,2]. COPD has become the third-leading cause of death worldwide, with a high incidence and disability rate, and it is accompanied by significant economic and social pressure[1,3]. Traditional Chinese medicine (TCM)therapies have been widely used for stable COPD with no or mild side effects and are gaining increasing attention for their significant effects. Bufei Yishen formula (BYF), a Chinese herbal formula, has shown good efficacy on COPD clinical symptoms including reducing the frequency of acute exacerbation by our previous studies[4]. However, the complex ingredients of BYF makes it difficult for elucidating the mechanisms involved and hinders the international promotion. Therefore, five effective components were identified from herbal medicines of BYF based on vivo experiments, combined in a fixed ratio as the effective-component compatibility of BYF $\otimes$ (ECC-BYF $\otimes)$, which suppressed the inflammation by regulating p65, JNK, and p38 in COPD[5]. 
Cell senescence in COPD, particularly in alveolar and airway epithelial cells, increases the risk of respiratory tract infection, progressive emphysema aggravation, and may lead to airway remodeling, which can be induced by extracellular or intracellular stimuli such as telomere attrition (replicative senescence), irreparable DNA damage, mitochondrial dysfunction, and oxidative stress (excessive senescence)[2,6-11]. Cell senescence is always accompanied by a complex phenotype, such as altered cell morphology, cell cycle arrest, increased senescence-associated $\beta$-galactosidase (SA- $\beta$-gal) and the senescence-associated secretory phenotype (SASP), which is primarily mediated by the $p 53$ or p16/p21 pathway[11]. Mitophagy is a type of elimination of irreversibly damaged mitochondria that helps to slow cell senescence. It is regulated by PTEN-induced putative kinase 1 (PINK1) and Parkin (PARK) 2. Studies have shown that oxidative stress may result in insufficient mitophagy by downregulating the expression of PINK1 and PARK2 $[8,12-15]$, elevated nuclear factor erythroid-2 related factor 2 (Nrf2) can alter the process[16, 17].

Therefore, we explored the effects of ECC-BYF $₫$ on COPD rats, and investigated the mechanism of ECCBYF $\triangle$ improved CSE-induced airway epithelial cell senescence by suppressing oxidative stress and subsequently enhancing insufficient mitophagy in the present study.

\section{Materials And Methods}

\section{Animals}

48 Sprague-Dawley rats (half male and half female, $200 \pm 20 \mathrm{~g}$ ) were purchased from the Laboratory Animal Center of Henan Province (Zhengzhou, China).

\section{Drugs}

The components of ECC-BYF $\otimes$ were purchased from Manster biotechnology co., LTD (Chengdu, China). NAC (Flumucil, as positive control in animal experiment) was purchased from Zambon Pharmaceutical Co., LTD, (Hainan, China). Luteolin (purity: $\geq 98 \%$, 491-70-3) was provided by Manster biotechnology co., LTD, (Chengdu, China).

\section{COPD model and administration}

After acclimatized for $7 \mathrm{~d}$, rats were randomized into the normal, model, ECC-BYF $\triangle$ and NAC groups (half male and half female in each group). The COPD model was established with tobacco smoke exposure and bacterial infection from week 1 to week 8 . Briefly, the model rats were exposed to tobacco smoke (smoke concentrations, $3000 \pm 500$ ppm, $1.0 \mathrm{mg}$ of nicotine, $11 \mathrm{mg}$ CO and $10 \mathrm{mg}$ of tar per cigarette; Hongqiqu ${ }^{\circledR}$ filter cigarettes, Zhengzhou, China) $40 \mathrm{~min}$, twice a day, and Klebsiella pneumoniae $(0.1 \mathrm{ml}$, $6 \times 10^{8} \mathrm{CFU} / \mathrm{mL}$; Bacterial strain: 46114; National Center for Medical Culture Collection, Beijing, China) for once every 5 days[18].

From 9 to 16 weeks, the rats of treatment groups were orally gavaged with ECC-BYF $\otimes$ (dosage: 6.48 $\mathrm{mg} / \mathrm{kg}, \mathrm{q} . \mathrm{d}$ ) or NAC (dosage: $54 \mathrm{mg} / \mathrm{kg}, \mathrm{q} . \mathrm{d}$ ). Meanwhile, normal rats were orally gavaged with saline 
once a day. The dosage of ECC-BYF $\triangle$ and NAC were decided and adjusted weekly by the following formula: $\mathrm{D}_{\text {rat }}=\mathrm{D}_{\text {human }} \times\left(\mathrm{K}_{\text {rat }} / \mathrm{K}_{\text {human }}\right) \times\left(\mathrm{W}_{\text {rat }} / \mathrm{W}_{\text {human }}\right)^{2 / 3}$ (D: dosage; $\mathrm{K}$ : body shape index; $\mathrm{W}$ : body weight). At week 16, the rats were sacrificed and samples were obtained.

\section{Pulmonary function analysis}

Pulmonary function was measured in unrestrained rats with Whole Body Plethysmograph (WBP) system (Buxco Inc., Wilmington, NC, USA) every 4 weeks. The relevant continuous ventilatory parameters, including Tidal volume $\left(\mathrm{V}_{\mathrm{T}}\right)$, minute volume $(\mathrm{MV})$, peak expiratory flow $(\mathrm{PEF})$, and expiratory flow at $50 \%$ tidal volume (EF50) were calculated meanwhile. A FinePointe ${ }^{T M}$ pulmonary function test system (Buxco Inc, USA) was used to measure the parameters of forced vital capacity (FVC) and forced expiratory volume at $0.3 \mathrm{~s}$ (FEV 0.3).

\section{Histopathology analysis}

The lung tissues were sliced into $3-4 \mathrm{~mm}$ sections, fixed in $10 \%$ paraformaldehyde solution for $72 \mathrm{~h}$, and then mounted in paraffin-embedded 3 micron sections. The sections were stained with hematoxylin-eosin $(\mathrm{H} \& E)$ staining. Whereafter, the stained tissue sections were taken utilizing optical microscopy and a photographic system (Olympus Optical Co., Ltd., Japan), and six images were taken for each section. And the alveolar mean linear intercept $(\mathrm{MLI}, \mu \mathrm{m})$ and the mean alveolar number $\left(\mathrm{MAN}, / \mathrm{mm}^{2}\right)$ were counted with the counting tool of Adobe Photoshop CC software to evaluate the degree of emphysema.

\section{Kit analysis}

The concentration of tumor necrosis factor-a (TNF-a), interleukin (IL)- 6 and matrix metalloproteinase (MMP)-9 in bronchoalveolar lavage fluid (BALF) were quantified by enzyme-linked immunosorbent assay (ELISA) kits (Boster Bio-Engineering Co., Ltd., Wuhan, China). Meanwhile, total superoxide dismutase (TSOD), malondialdehyde (MDA)] and glutathione peroxidase (GSH-PX) in lung tissue or cells were measured with kits (Elabscience, Wuhan, China) according to the manufacturer's protocol.

\section{Immunofluorescence analysis}

The expression of p21 in lung, especially in bronchus was detected by immunofluorescence. After dewaxing and dehydration treatment, the lung tissue slices were added with a 5\% BSA for $30 \mathrm{~min}$, and then anti-p21 antibody was incubated (1:1000 dilution; Cell signaling technology) overnight at $4{ }^{\circ} \mathrm{C}$. On the following day, slices were incubated with Cy3-conjugated affinipure goat Anti-Rabbit lgG $(\mathrm{H}+\mathrm{L})$ (proteintech, Wuhan, China) for 50 min. At least six images were randomly taken for each section and the red staining area was calculated by CaseViewer software.

Moreover, BEAS-2B cells cultured in 24-well culture slides were fixed with 4\% paraformaldehyde for 15 min and blocked with sheep serum protein mixed with $0.3 \%$ TritonX-100 for $2 \mathrm{~h}$ at room temperature. The primary antibody LC3B (GTX17380, Gene Tex), TOM20 (11802-1-AP, proteintech) and the secondary antibody were incubated according to the manufacturer' instruction. Finally, confocal laser scanning microscopy was used to picture and assess the mitophagy. 


\section{Preparation of cigarette extract}

The mainstream cigarette smoke was sucked into $50 \mathrm{ml}$ syringe, and then slowly injected into the serumfree DMEM medium. The optical density at $320 \mathrm{~nm}$ wavelength was measured with spectrophotometer, and adjusted to 1.8-2.0 with DMEM medium. Subsequently, the prepared CSE solution was filtered through a filter $(0.22 \mu \mathrm{M})$ to obtain $100 \%$ CSE solution.

\section{Cell culture and treatment}

The BEAS-2B (ATCC), a human bronchial cell line, were cultured in DMEM culture medium (Solarbio, Beijing, China) with $10 \%$ fetal bovine serum (FBS). In short, after being maintained with FBS-free medium

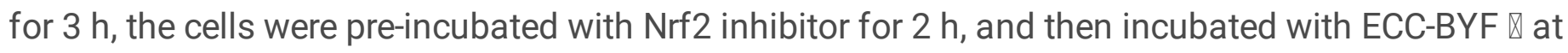
different concentrations $(35 \mu \mathrm{g} / \mathrm{ml}, 17.5 \mu \mathrm{g} / \mathrm{ml}, 8.75 \mu \mathrm{g} / \mathrm{ml})$ for $3 \mathrm{~h}, 10 \%$ cigarette smoke extract (CSE) was added subsequently. The cells were collected after $6 \mathrm{~h}$.

\section{SA- $\beta$-Gal Staining}

The experiment of SA- $\beta-G$ al staining was carried out following the manufacturer's instruction (G1580, Solarbio). The proportion of stained cells to total BEAS-2B cells was calculated.

\section{Electron microscopy}

BEAS-2B cells were fixed with $2.5 \%$ glutaraldehyde (P1126S, Solarbio, Beijing, China) overnight at $4{ }^{\circ} \mathrm{C}$. Samples formulation and electron microscope photography were completed by the electron microscope Center of Henan University of Traditional Chinese Medicine. Mitochondria and autophagosomes were assessed.

\section{Western Blotting}

The cells were lysed in a RIPA lysis mixing protease inhibitor and phosphatase inhibitor. The concentration of protein was determined by BCA protein assay kit (PC0020, Solarbio, Beijing, China). The protein samples were separated in 10\% SDS-PAGE and transferred to polyvinylidene difluoride (PVDF) membranes. The membranes were blocked with $5 \%$ skimmed milk in TBST at room temperature for $1 \mathrm{~h}$ and incubated with the primary antibodies overnight at $4{ }^{\circ} \mathrm{C}$. The specific primary antibodies were $\beta$-actin (1:1000 diluted; proteintech), p21 (1:1000 diluted; CST), p16(1:1000 diluted; proteintech), PINK1 (1:1000 diluted; proteintech), PARK2 (1:1000 diluted; proteintech), Mfn2 (1:1000 diluted; CST), Drp1 (1:1000 diluted; CST), Nrf2 (1:1000 diluted; Gene Tex), HO-1 (1:1000 diluted; CST). Subsequently, the membranes were incubated with secondary antibodies of HRP-conjugated goat anti-mouse and anti-rabbit (1:5000 diluted, proteintech) for $1 \mathrm{~h}$ at room temperature. After Washed three times with TBST, the bands were visualized with enhanced chemiluminescence ECL reagent. The interest protein bands intensities were adjusted with $\beta$-actin control intensities.

\section{Statistic Analysis}


All data were processed by SPSS 22.0 software and graphed with Graphpad prism 10.0. Data were presented as the means \pm standard deviation. Statistically significant differences were assessed by oneway ANOVA followed by the Tukey's test where appropriate. Values of $P<0.05$ were considered statistically significant.

\section{Results}

\section{Effect of ECC-BYF $\triangle$ on pulmonary function and histopathological changes of COPD rats}

The severity of COPD is determined by pulmonary function and histopathological changes. $V_{T}, M V, P E F$, and EF50 were all significantly lower in COPD rats, as were FVC and FEV 0.3, as shown in Figure 1. Treatment with ECC-BYF III and N-Acetylcysteine (NAC) improved lung function significantly. Figure 2 depicts the histopathological changes in COPD, including alveolar and bronchial thickening, emphysema, and inflammatory cell infiltration. Quantitative analysis of MAN and MLI showed that increased MLI was accompanied by decreased MAN. Treatment with ECC-BYF $\triangle$ and NAC could effectively rescue the histopathological changes above. These data suggested that ECC-BYF III could attenuate COPD rats.

\section{Effect of ECC-BYF $₫$ on cell senescence-associated secretory phenotype in COPD rats}

Lung cell senescence results in the release of SASP such as the inflammatory cytokines (IL-1 $\beta$, IL-6, and TNF-a), chemokines (CXCL-1, CXCL-8, and CCL-2), growth factors, proteases (MMP-2, MMP-9). It also induces cell cycle arrest, decreased antioxidant capacity (T-SOD lower), and damaged-associated molecular patterns $[6,11,19,20]$. MDA activity, MMP-9 level, and TNF- $a$ and IL-6 expression were all suppressed after ECC-BYF III and NAC administration, whereas T-SOD expression was increased (Figure3A-E). According to the immunofluorescence results, ECC-BYF III treatment significantly suppressed the expression of p21, a cyclin-dependent kinase inhibitor, indicating cell senescence with persistently elevated expression (Figure3F-G). These findings suggested that ECC-BYF III treatment improved a number of cell senescence-associated secretory phenotypes in the lungs of COPD rats.

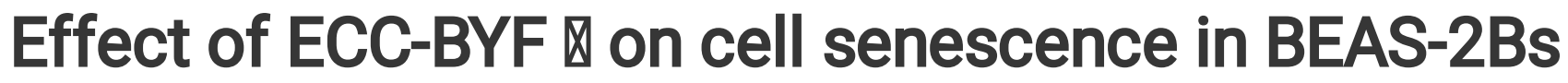 induced by CSE}

Previous research has found a high level of p21 expression in lung tissue, particularly in the airways of COPD rats, and we know that cigarette smoke exposure is a major factor that causes elevated p21 expression, as well as airway epithelial cell senescence and COPD airway epithelial dysfunction. The expressions of $\mathrm{p} 21, \mathrm{p} 16$, and SA- $\beta$-Gal were detected in CSE-induced BEAS-2Bs to determine the effect of ECC-BYF III on cell senescence. We found that 10\% CSE exposure increased the expression of p21 and $p 16$, and SA- $\beta$-Gal staining. ECC-BYF $\otimes$ treatment demonstrated a significant decrease in the expression of 
p21 and p16 and the number of SA-B-Gal-stained cells (Figure 4). These findings suggested that ECC-BYF $\nabla$ effectively suppressed CSE-induced airway epithelial cell senescence.

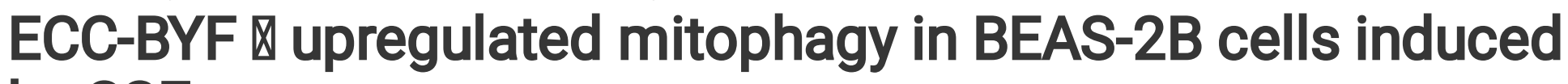 by CSE}

Mitophagy deficiency is a critical inducer of the accumulation of damaged mitochondrial and subsequent

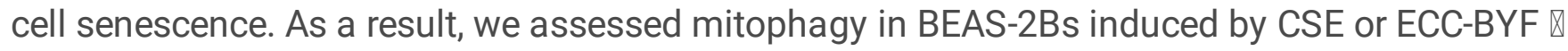
treatment. We found that ECC-BYF $\otimes$ could significantly increase the expression of PINK1, PARK2, and intensified the co-localization of TOM20-labeled mitochondria, as shown by the yellow dots and LC3B (green dots) in figure 5B. Furthermore, we found that ECC-BYF $\otimes$ treatment obviously improved the swelling of mitochondria, decreased the number of damaged mitochondria, and increased the quantity of autophagosomes and lysosomes. Meanwhile, the expression of Mfn2 increased and Drp1 decreased. Taken together, ECC-BYF $\triangle$ exhibited effective role in enhancing mitophagy, subsequently eliminating damaged mitochondria, and improving mitochondrial function (Figure 5).

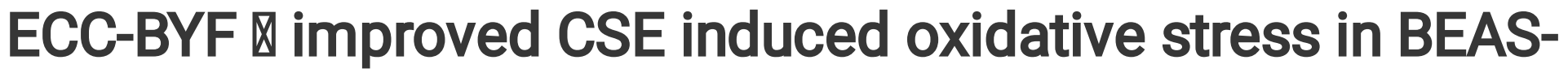 2Bs}

CSE-induced insufficient mitophagy and abundant damaged mitochondria are frequently caused by oxidative stress [21]. After CSE exposure, reactive oxygen species (ROS) increased while the activities of T-SOD and GSH-PX were down-regulated in BEAS-2Bs. ROS was reduced by ECC-BYF III treatment, whereas the activity of T-SOD and GSH-PX was increased in BEAS-2Bs (Figure 6A). Furthermore, we discovered that ECC-BYF III treatment significantly increased Nrf2 and HO-1 (Figure 6B). The findings suggested that ECC-BYF III could activate Nrf2 and prevent CSE-induced oxidative stress.

\section{Involvement of NIf2 signal in ECC-BYF Q ${ }^{\prime} s$ anti- senescence}

To investigate the role of Nrf2 in the anti-senescence effect of ECC-BYF III, we treated BEAS-2Bs with Nrf2 inhibitors and/or ECC-BYF III. We discovered that co-treatment with Luteolin (Nrf2 inhibitor) inhibited the effects of ECC-BYF III, which could be reflected by the down-regulation of Nrf2 and HO-1 and higher ROS activity, as well as inhibiting the activation of related proteins (PINK1, PARK2, Mfn2) and significantly upregulating the expression of Drp1. Luteolin eventually inhibited ECC-BYF III's ability to reduce the expression of p16 and p21 (Figure 7). All data demonstrated that ECC-BYF $\triangle$ may play a role in ameliorating cell senescence through inhibiting oxidative stress and mitochondrial function through Nrf2 signaling.

\section{Discussion}

COPD is a major social problem that endangers public health because of its high morbidity and mortality rates. Traditional Chinese medicine is widely acknowledged for its efficacy in the treatment of COPD. The original BYF, which included twelve Chinese medicines, had a positive effect on COPD clinical

symptoms[4, 22]. However, the complexity of the ingredients makes elucidating the mechanisms involved 
difficult. The effective compounds were identified from BYF and combined with a fixed ratio to produce the ECC-BYF III. 20-S-ginsenoside Rh1, astragaloside IV, icariin, nobiletin, and paeonol are among the five compounds found in ECC-BYF III[5, 23]. ECC-BYF III effectively rescued pulmonary function, lung histopathological changes, and cell senescence-associated secretory phenotype in COPD rats in this study. Furthermore, by inhibiting oxidative stress and promoting insufficient mitophagy, ECC-BYF III may be able to alleviate CSE-induced airway epithelial cell senescence.

Lung senescence, mainly including alveolar and airway epithelial cell senescence, participates in the pathogenesis of $\operatorname{COPD}[2,6]$. In COPD, cigarette smoke is a major driver of cell senescence. The accumulation of senescent cells in lung contributes to the acquisition of SASP, and senescence increases susceptibility to infection, airway remodeling and exacerbation of COPD-emphysema[8, 10, 24]. The SASP takes the form of cell cycle arrest, the release of inflammatory cytokines, proteases, and the reduction of antioxidant capacity[25, 26]. Indeed, our studies found that the SASP occurred in COPD rats, such as increased p21, TNF-a, IL-6, MMP-9 and MDA activity and T-SOD level decreased significantly in lung or BALF. ECC-BYF $\otimes$ suppressed pulmonary function reduction and histopathological changes, meanwhile, improved SASP in COPD rats. Thus, we conclude that ECC-BYF $\triangle$ exerted distinct effect on COPD rats through ameliorating the airway epithelial cell senescence and the SASP.

The first barrier against external stimuli are airway epithelial cells. CS typically promotes airway epithelial cell senescence, with elevated p16, p21, and SA- $\beta$-Gal expression[27, 28]. Our findings showed that ECCBYF III can prevent CSE-induced airway epithelial cell senescence. Excessive oxidants/ROS produced by CS exposure can damage biological macromolecules and cause mitochondrial dysfunction[29, 30]. Given that mitochondria are the pivotal hub of energy production and the producer of ROS, substantial studies have proved the importance of mitochondrial dysfunction in CS-induced cell senescence[12, 31]. Mitophagy is a special form of autophagy where damaged mitochondria are eliminated to maintain the mitochondrial function. Insufficient mitophagy leads to the accumulation of damaged mitochondria, and thus cause the deficiency of energy, which impels cell senescence[28, 32, 33]. There are evidence suggesting that PINK1 and PARK2 recruitment are required for mitophagy to ameliorate CSE-induced cell senescence, PINK1 knockdown noticeably reduced the expression of PARK2, and PARK2 knockdown could elevate the expression of p21 and SA- $\beta$-Gal staining in response to CSE exposure[12, 15, 28]. In this study, we found ECC-BYF $\triangle$ could inhibit the production of ROS induced by CSE, and enhance mitophagy by increasing the levels of PINK1 and PARK2. Aravamudan Bharathi et al pointed out that CSE induced mitochondrial fragmentation and dysfunction as marked by decreasing Mfn2 and increasing Drp1[34]. Treatment with ECC-BYF $\otimes$ could increase the expression of Mfn2 and reduce Drp1, also alleviate the swelling and cristae disruption of mitochondria in CSE-induced airway epithelial cells with the increase of autophagosomes and lysosomes. This is the result of ECC-BYF III enhancing mitophagy and improving mitochondrial function. As a result, we hypothesized that ECC-BYF III reduced cell senescence by increasing the activity of PINK1 and PARK2. Since Nrf2 is a common anti-oxidant factor and activation of Nrf2 can protect cells from mitophagy deficiency caused by oxidative stress[16, 35, 36], we administered cells with Nrf2 inhibitor and discovered that Nrf2 inhibitor suppressed the inhibition effect of ECC-BYF III on ROS production, as well as mitophagy deficiency and cell senescence. Hence, Targeting the Nrf2 
pathway to inhibit oxidative stress and intensify mitophagy is the potential mechanism for ECC-BYF $\otimes$ to ameliorate cell senescence.

\section{Conclusion}

ECC-BYF $\triangle$ was effective in the treatment of COPD rats through ameliorating airway epithelial cell senescence. The effect was due to inhibition of oxidative stress and enhancement of mitophagy by Nrf2/PINK1.

\section{Abbreviations}

COPD, chronic obstructive pulmonary disease; CSE, Cigarette smoke extract; Drp1, dynamin-related

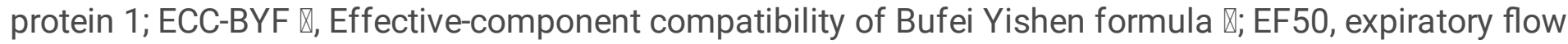
at $50 \%$ tidal volume; FEV 0.3 , forced expiratory volume at $0.3 \mathrm{~s}$; FVC, forced vital capacity; GSH-PX, glutathione peroxidase; IL-6, interleukin 6; MAN, mean alveolar number; MDA, malondialdehyde; Mfn, mitofusin; MLI, mean linear intercept; MMP-9, matrix metalloproteinase; MV, minute volume; NAC, NAcetylcysteine; Nrf2, nuclear factor erythroid-2 related factor 2; PARK, Parkin; PEF, peak expiratory; PINK1, PTEN-induced putative kinase 1; ROS, reactive oxygen species; SASP, senescence-associated secretory phenotype; SA- $\beta$-Gal, senescence-associated $\beta$-galactosidase; TNF- $\alpha$, tumor necrosis factor- $\alpha$; T-SOD, total superoxide dismutase; $\mathrm{V}_{\mathrm{T}}$, tidal volume

\section{Declarations}

\section{Ethics and consent to participate}

All animal procedures were approved by the Experimental Animal Care and Ethics Committee of the First Affiliated Hospital, Henan University of Traditional Chinese Medicine, Zhengzhou, China (DWLL201903210). All methods were conducted in accordance with the ARRIVE guidelines.

\section{Consent for publication}

Not applicable.

\section{Availability of data and material}

The datasets used and/or analysed during this study are available from the corresponding author on reasonable request.

\section{Conflict of interests}

The authors declare that they have no competing interests concerned with this article.

\section{Founding}


This research was supported by National Natural Science Fund of China (No. 81973822, No. 82104662) and Key scientific research projects of Henan Province Colleges and Universities (No. 21B360004).

Author contribution

JSL, PZ and YGT designed this study. MYL, YQQ, KCL and XFL performed the animal experiments; MYL and PZ conceived and performed the cell experiments; MYL, YQQ, PZ and BGO drafted and revised the manuscript. MYL and YQQ contributed to the data analysis. And JSL and PZ supervised this study. All authors have read and approved the manuscript.

Acknowledgements

Not applicable.

\section{References}

1. 2021 GOLD Reports - Global Initiative for Chronic Obstructive Lung Disease - GOLD. In., vol. 2021; 2021.

2. Agusti A, Hogg JC: Update on the Pathogenesis of Chronic Obstructive Pulmonary Disease. N Engl J Med 2019, 381(13):1248-1256.

3. Lopez-Campos JL, Tan W, Soriano JB: Global burden of COPD. RESPIROLOGY 2016, 21(1):14-23.

4. Li JS, Li SY, Xie Y, Yu XQ, Wang MH, Sun ZK, Ma LJ, Jia XH, Zhang HL, Xu JP et al: The effective evaluation on symptoms and quality of life of chronic obstructive pulmonary disease patients treated by comprehensive therapy based on traditional Chinese medicine patterns. COMPLEMENT THER MED 2013, 21(6):595-602.

5. Li J, Xie Y, Zhao P, Qin Y, Oliver BG, Tian Y, Li S, Wang M, Liu X: A chinese herbal formula ameliorates COPD by inhibiting the inflammatory response via downregulation of p65, JNK, and p38. PHYTOMEDICINE 2021, 83:153475.

6. Barnes PJ, Baker J, Donnelly LE: Cellular Senescence as a Mechanism and Target in Chronic Lung Diseases. Am J Respir Crit Care Med 2019, 200(5):556-564.

7. Munoz-Espin D, Serrano M: Cellular senescence: from physiology to pathology. Nat Rev Mol Cell Biol 2014, 15(7):482-496.

8. Vij N, Chandramani-Shivalingappa P, Van Westphal C, Hole R, Bodas M: Cigarette smoke-induced autophagy impairment accelerates lung aging, COPD-emphysema exacerbations and pathogenesis. Am J Physiol Cell Physiol 2018, 314(1):C73-C87.

9. Birch J, Anderson RK, Correia-Melo C, Jurk D, Hewitt G, Marques FM, Green NJ, Moisey E, Birrell MA, Belvisi MG et al: DNA damage response at telomeres contributes to lung aging and chronic obstructive pulmonary disease. Am J Physiol Lung Cell Mol Physiol 2015, 309(10):1124-1137.

10. Naikawadi RP, Disayabutr S, Mallavia B, Donne ML, Green G, La JL, Rock JR, Looney MR, Wolters PJ: Telomere dysfunction in alveolar epithelial cells causes lung remodeling and fibrosis. JCl Insight 
2016, 1(14):86704.

11. Coppe JP, Desprez PY, Krtolica A, Campisi J: The senescence-associated secretory phenotype: the dark side of tumor suppression. Annu Rev Pathol 2010, 5:99-118.

12. Yoo SM, Jung YK: A Molecular Approach to Mitophagy and Mitochondrial Dynamics. MOL CELLS 2018, 41(1):18-26.

13. Sundar IK, Maremanda KP, Rahman I: Mitochondrial dysfunction is associated with Miro1 reduction in lung epithelial cells by cigarette smoke. TOXICOL LETT 2019, 317:92-101.

14. Chen F, Liu Y, Wong NK, Xiao J, So KF: Oxidative Stress in Stem Cell Aging. CELL TRANSPLANT 2017, 26(9):1483-1495.

15. Sarraf SA, Sideris DP, Giagtzoglou N, Ni L, Kankel MW, Sen A, Bochicchio LE, Huang CH, Nussenzweig SC, Worley SH et al: PINK1/Parkin Influences Cell Cycle by Sequestering TBK1 at Damaged Mitochondria, Inhibiting Mitosis. CELL REP 2019, 29(1):225-235.

16. Murata $\mathrm{H}$, Takamatsu H, Liu S, Kataoka K, Huh NH, Sakaguchi M: NRF2 Regulates PINK1 Expression under Oxidative Stress Conditions. PLOS ONE 2015, 10(11):e142438.

17. Silva-Palacios A, Ostolga-Chavarria M, Zazueta C, Konigsberg M: Nrf2: Molecular and epigenetic regulation during aging. AGEING RES REV2018, 47:31-40.

18. Li Y, Li SY, Li JS, Deng L, Tian YG, Jiang SL, Wang Y, Wang YY: A rat model for stable chronic obstructive pulmonary disease induced by cigarette smoke inhalation and repetitive bacterial infection. BIOL PHARM BULL 2012, 35(10):1752-1760.

19. Tai H, Wang Z, Gong H, Han X, Zhou J, Wang X, Wei X, Ding Y, Huang N, Qin J et al: Autophagy impairment with lysosomal and mitochondrial dysfunction is an important characteristic of oxidative stress-induced senescence. AUTOPHAGY 2017, 13(1):99-113.

20. Dodig S, Cepelak I, Pavic I: Hallmarks of senescence and aging. Biochem Med (Zagreb) 2019, 29(3):30501.

21. Zhang Y, Xi X, Mei Y, Zhao X, Zhou L, Ma M, Liu S, Zha X, Yang Y: High-glucose induces retinal pigment epithelium mitochondrial pathways of apoptosis and inhibits mitophagy by regulating ROS/PINK1/Parkin signal pathway. BIOMED PHARMACOTHER 2019, 111:1315-1325.

22. Li SY, Li JS, Wang MH, Xie Y, Yu XQ, Sun ZK, Ma LJ, Zhang W, Zhang HL, Cao F et al: Effects of comprehensive therapy based on traditional Chinese medicine patterns in stable chronic obstructive pulmonary disease: a four-center, open-label, randomized, controlled study. BMC Complement Altern Med 2012, 12:197.

23. Li J, Ma J, Tian Y, Zhao P, Liu X, Dong H, Zheng W, Feng S, Zhang L, Wu M et al: Effective-component compatibility of Bufei Yishen formula II inhibits mucus hypersecretion of chronic obstructive pulmonary disease rats by regulating EGFR/PI3K/mTOR signaling. J ETHNOPHARMACOL 2020, 257:112796.

24. Adnot S, Amsellem V, Boyer L, Marcos E, Saker M, Houssaini A, Kebe K, Dagouassat M, Lipskaia L, Boczkowski J: Telomere Dysfunction and Cell Senescence in Chronic Lung Diseases: Therapeutic Potential. Pharmacol Ther 2015, 153:125-134. 
25. Houssaini A, Breau M, Kebe K, Abid S, Marcos E, Lipskaia L, Rideau D, Parpaleix A, Huang J, Amsellem $\mathrm{V}$ et al: $\mathrm{mTOR}$ pathway activation drives lung cell senescence and emphysema. JCl Insight 2018, 3(3).

26. Baker JR, Donnelly LE, Barnes PJ: Senotherapy: A New Horizon for COPD Therapy. CHEST 2020, 158(2):562-570.

27. Wu N, Yang D, Wu Z, Yan M, Zhang P, Liu Y: Insulin in high concentration recede cigarette smoke extract induced cellular senescence of airway epithelial cell through autophagy pathway. Biochem Biophys Res Commun 2019, 509(2):498-505.

28. Ito S, Araya J, Kurita Y, Kobayashi K, Takasaka N, Yoshida M, Hara H, Minagawa S, Wakui H, Fujii S et al: PARK2-mediated mitophagy is involved in regulation of HBEC senescence in COPD pathogenesis. AUTOPHAGY 2015, 11(3):547-559.

29. Fischer BM, Voynow JA, Ghio AJ: COPD: balancing oxidants and antioxidants. Int J Chron Obstruct Pulmon Dis 2015, 10:261-276.

30. Chen D, Kerr C: The Epigenetics of Stem Cell Aging Comes of Age. TRENDS CELL BIOL 2019, 29(7):563-568.

31. Korolchuk VI, Miwa S, Carroll B, von Zglinicki T: Mitochondria in Cell Senescence: Is Mitophagy the Weakest Link? EBIOMEDICINE 2017, 21:7-13.

32. Sachdeva K, Do DC, Zhang Y, Hu X, Chen J, Gao P: Environmental Exposures and Asthma Development: Autophagy, Mitophagy, and Cellular Senescence. FRONT IMMUNOL 2019, 10:2787.

33. Fujii S, Hara H, Araya J, Takasaka N, Kojima J, Ito S, Minagawa S, Yumino Y, Ishikawa T, Numata T et al: Insufficient autophagy promotes bronchial epithelial cell senescence in chronic obstructive pulmonary disease. ONCOIMMUNOLOGY 2012, 1(5):630-641.

34. Aravamudan B, Kiel A, Freeman M, Delmotte P, Thompson M, Vassallo R, Sieck GC, Pabelick CM, Prakash YS: Cigarette smoke-induced mitochondrial fragmentation and dysfunction in human airway smooth muscle. Am J Physiol Lung Cell Mol Physiol 2014, 306(9):840-854.

35. Dinkova-Kostova AT, Abramov AY: The emerging role of Nrf2 in mitochondrial function. Free Radic Biol Med 2015, 88(Pt B):179-188.

36. Xiao L, Xu X, Zhang F, Wang M, Xu Y, Tang D, Wang J, Qin Y, Liu Y, Tang C et al: The mitochondriatargeted antioxidant MitoQ ameliorated tubular injury mediated by mitophagy in diabetic kidney disease via Nrf2/PINK1. REDOX BIOL 2017, 11:297-311.

\section{Figures}



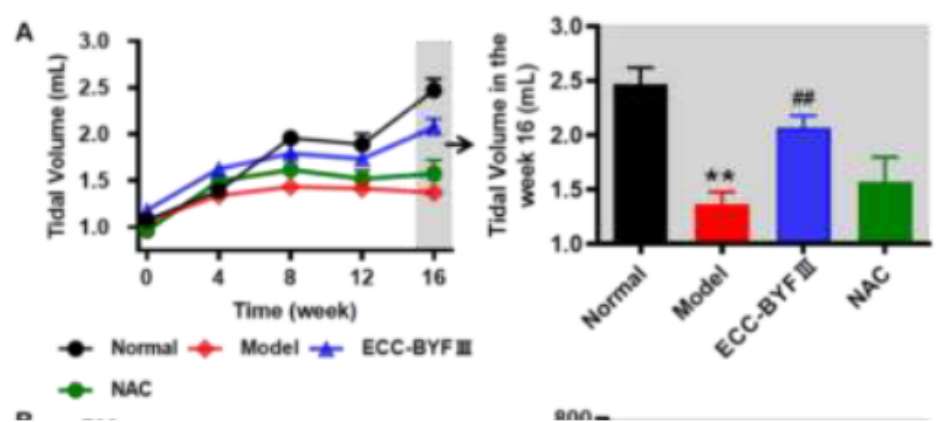

\section{Figure 1}

Effect of ECC-BYF $\otimes$ on pulmonary function of COPD rats. ECC-BYF $\otimes$ (dosage: $6.48 \mathrm{mg} / \mathrm{kg}, \mathrm{q} . \mathrm{d}$ ) or NAcetylcysteine (dosage: $54 \mathrm{mg} / \mathrm{kg}$, q.d) was given treatment groups by intragastric administration from 9 to 16 weeks. (A) Changes of $\mathrm{V}_{\mathrm{T}}$ in all groups. (B) Changes of MV in all groups. (C) Changes of PEF in all groups. (D) Changes of EF50 in all groups. (E) Changes of FEV0.3 in all groups. (F) Changes of FVC in all 
groups. All data are expressed as the mean $\pm \mathrm{SE},(\mathrm{n}=6) .{ }^{*} P<0.01$ vs. the normal group; ${ }^{\#} P<0.05$ vs. the model group, ${ }^{\#} P<0.01$ vs. the model group.

A

Normal

Model

ECC-BYF II

NAC
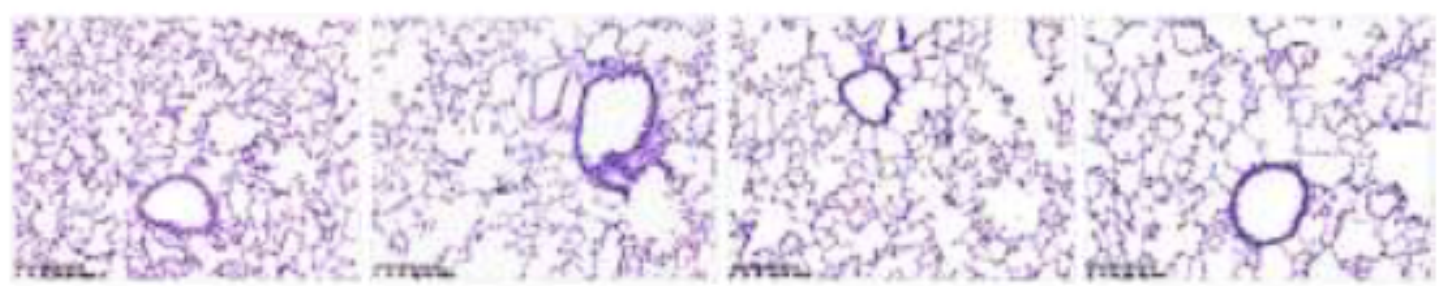

B
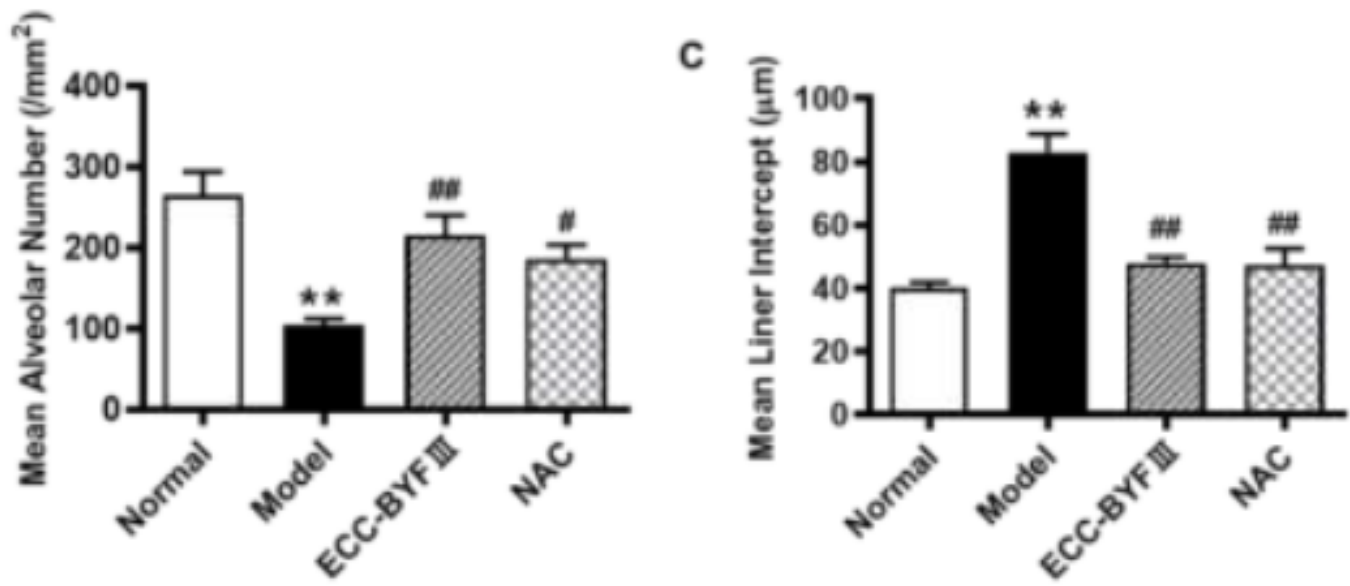

Figure 2

Effect of ECC-BYF $₫$ on histopathological changes of COPD rats. (A) H\&E staining of lungs from the different treatment groups $(\times 100)$. (B) Changes of MAN in different groups. (C) Changes of MLI in the different groups. The data are expressed as the mean $\pm \mathrm{SE},(\mathrm{n}=6) .{ }^{* *} P<0.01 \mathrm{vs}$. the normal group; ${ }^{\#} P<0.05$ vs. the model group, ${ }^{\#} P<0.01$ vs. the model group. 
A

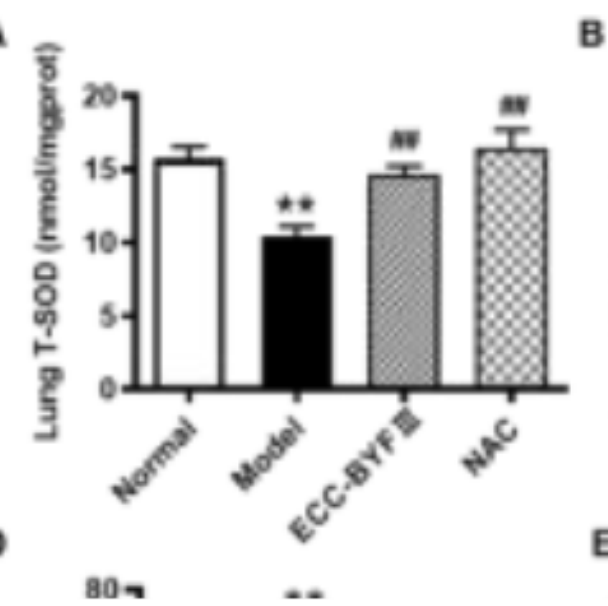

B

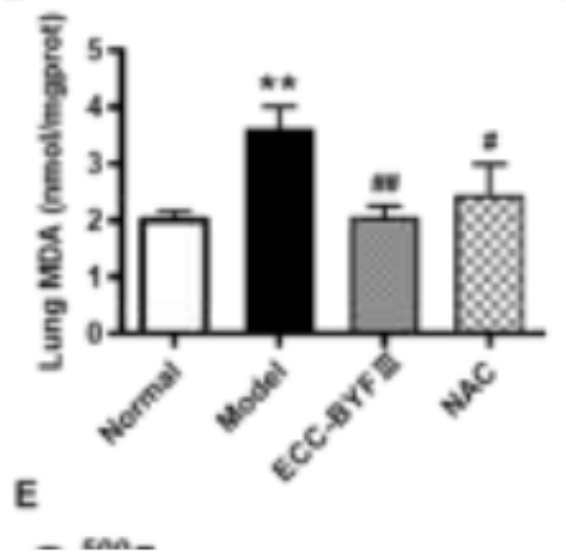

C

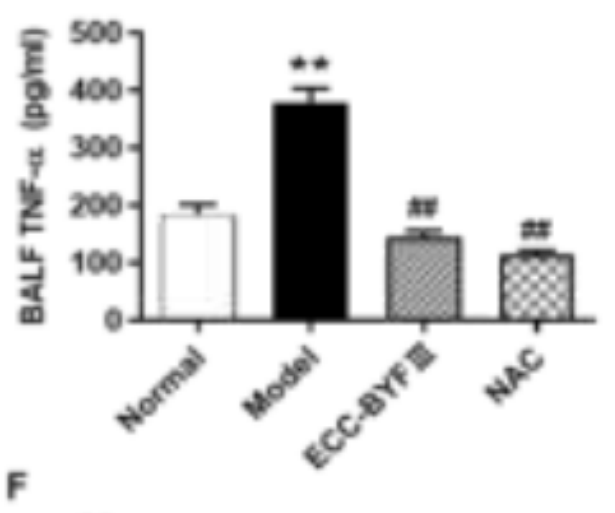

$=30$ 工

\section{Figure 3}

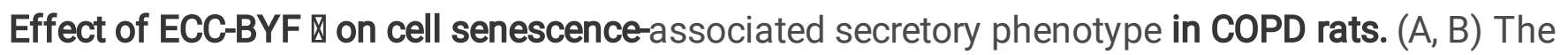
expression of T-SOD and MDA in lung tissue; (C, D) The expression of inflammation cytokines in BALF; (E) The expression of MMP-9 in BALF; (F) The expression of p21 positive area (\%). The data are expressed as the mean $\pm S E,(n=6)$. ${ }^{* *} P<0.01$ vs. the normal group, ${ }^{*} P<0.05$ vs. the normal group; ${ }^{\# \#} P<0.01$ vs. the model group, ${ }^{\#} P<0.05$ vs. the model group; (G) immunofluorescent staining of $p 21$, and red-stained represents the expression of $\mathrm{p} 21$, (magnification, $\times 200$ ). 
A
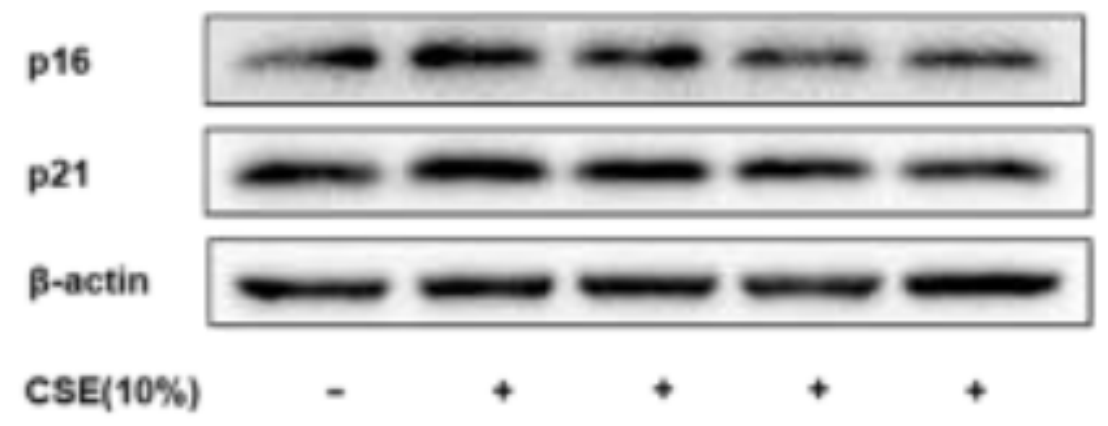

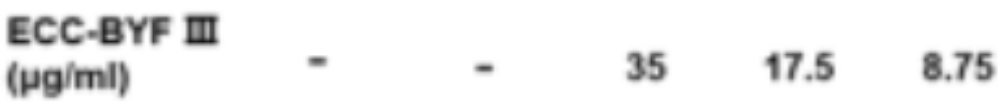

B

Normal

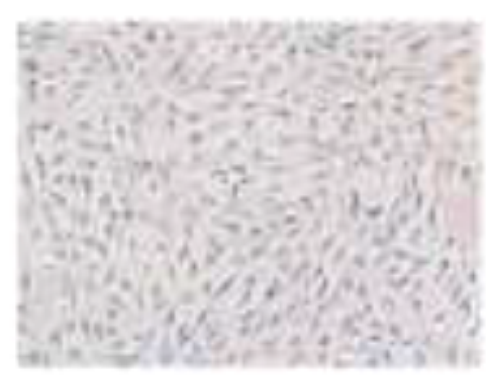

C

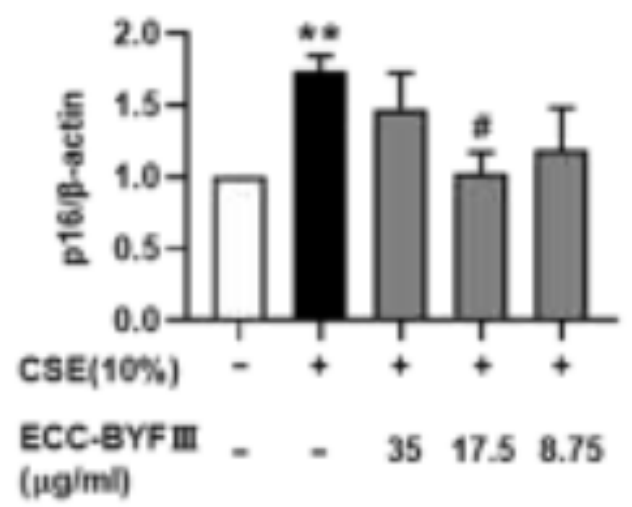

Model

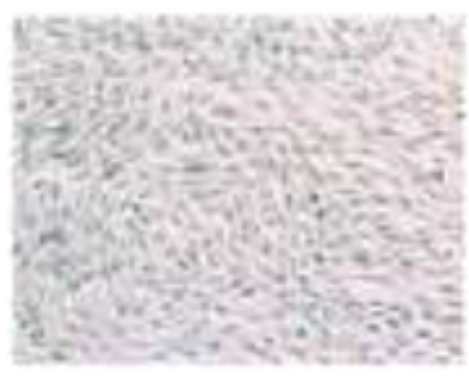

D

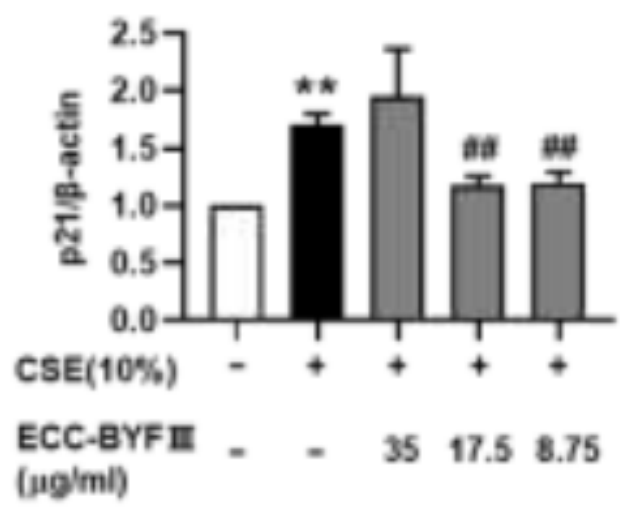

ECC-BYF III

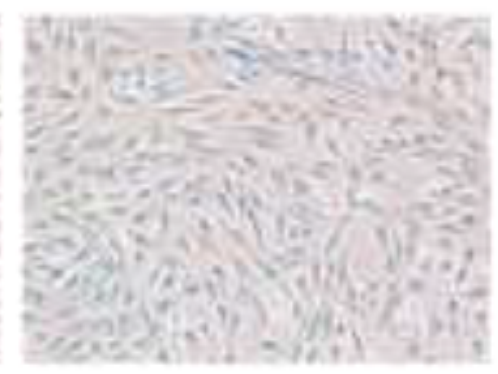

E

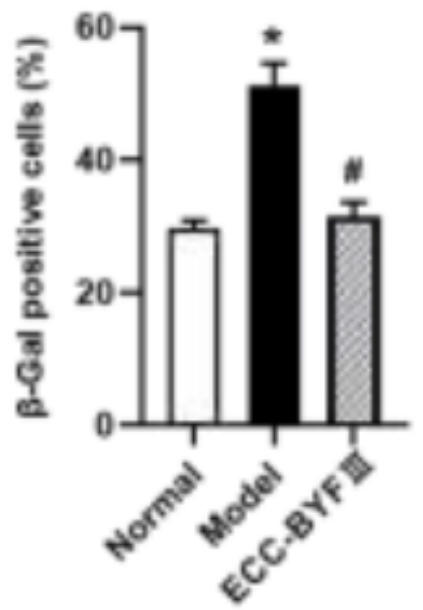

\section{Figure 4}

Effects of ECC-BYF $\mathbb{\Delta}$ on cells senescence in CSE-induced BEAS-2B. (A) The total protein expressions of p21 and p16; (B) SA- $\beta$-Gal staining of different groups; the level of (C) p16 (D) p21 and (E) a histogram of percentage of positive cells. The data are expressed as the mean $\pm S E,(n=3-6) .{ }^{* *} P<0.01$ vs. the normal group, ${ }^{*} P<0.05$ vs. the normal group; ${ }^{\#} P<0.01$ vs. the model group, ${ }^{\#} P<0.05$ vs. the model group. 
A

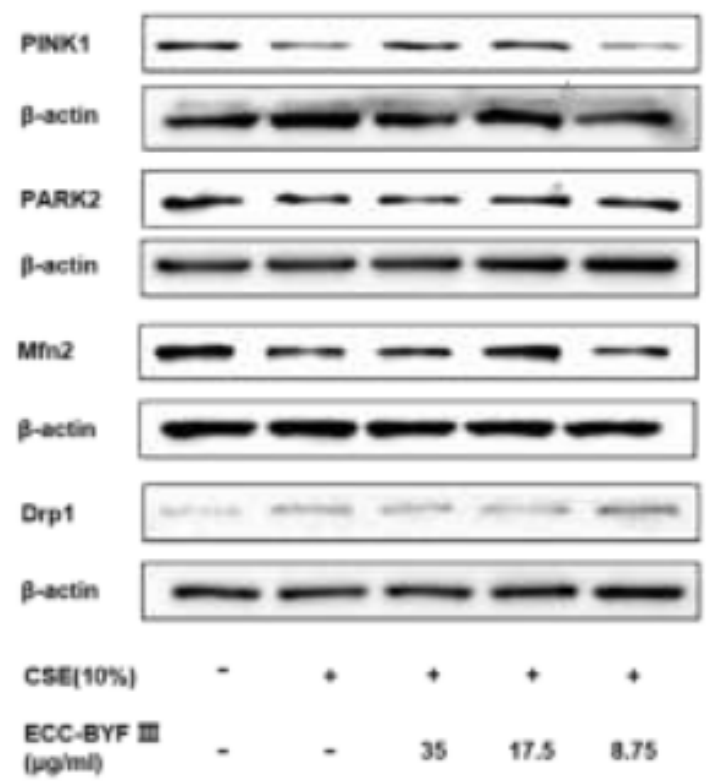

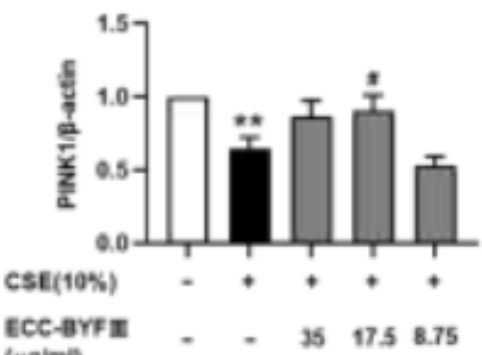

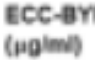

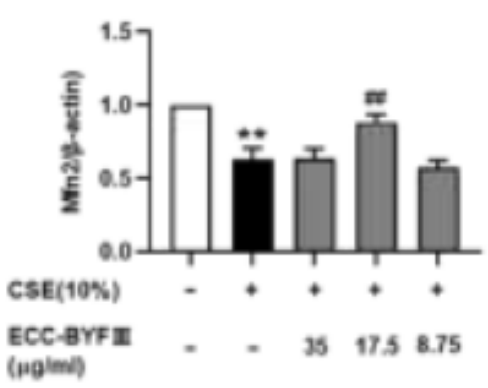

CSE(10\%)

ECC-AYFI (pgiml)

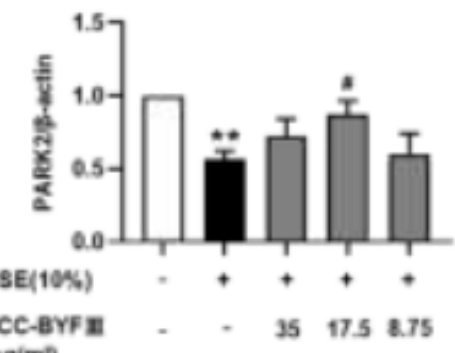

(ugimi)

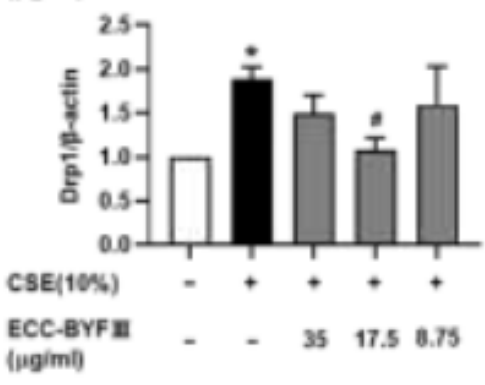

B

Normal

Mode!

ECC-BrF
$(17.5 \mu g i m i n)$

c

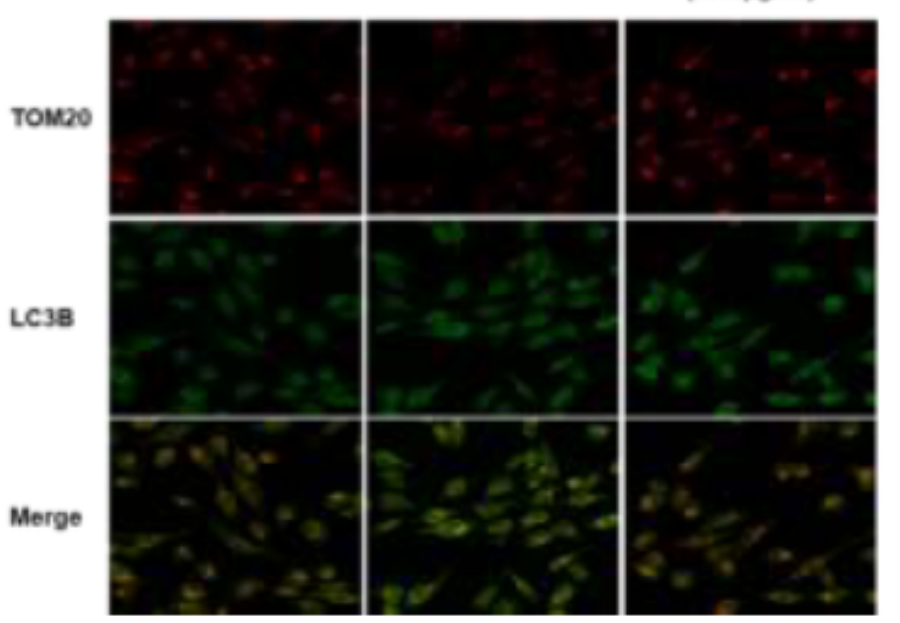

Normal

Model

ECC-BYF III
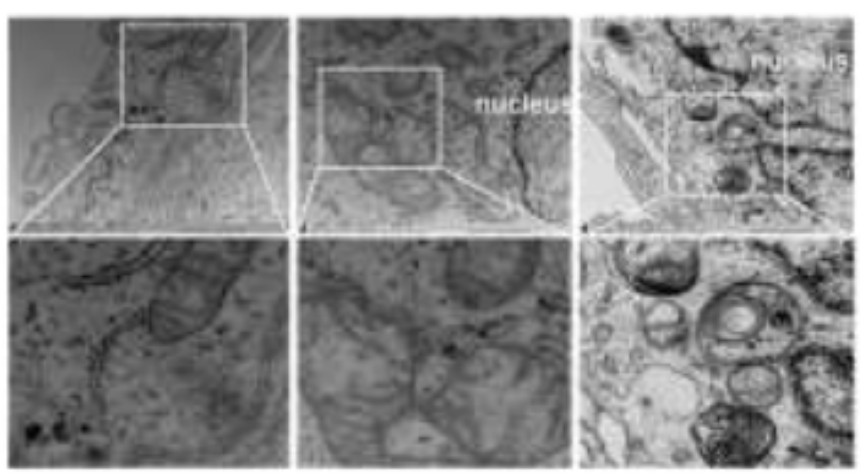

Figure 5

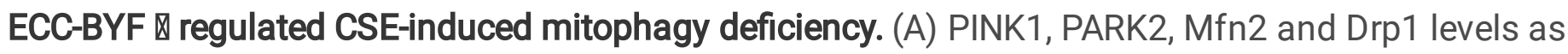
assessed by western blot; The data are expressed as the mean $\pm S E,(n=4)$. ${ }^{\star \star} P<0.01$ vs. the normal group, ${ }^{*} P<0.05$ vs. the normal group; ${ }^{\# \#} P<0.01$ vs. the model group, ${ }^{\#} P<0.05$ vs. the model group; (B) Immunofluorescent staining of the co-localization of TOM20 (yellow dots) and LC3B (green dots), (magnification, $\times 200$ ); (C) Electron microscopy detection of mitochondria and mitophagy in BEAS-2Bs (magnification, $\times 30000)$. 
A
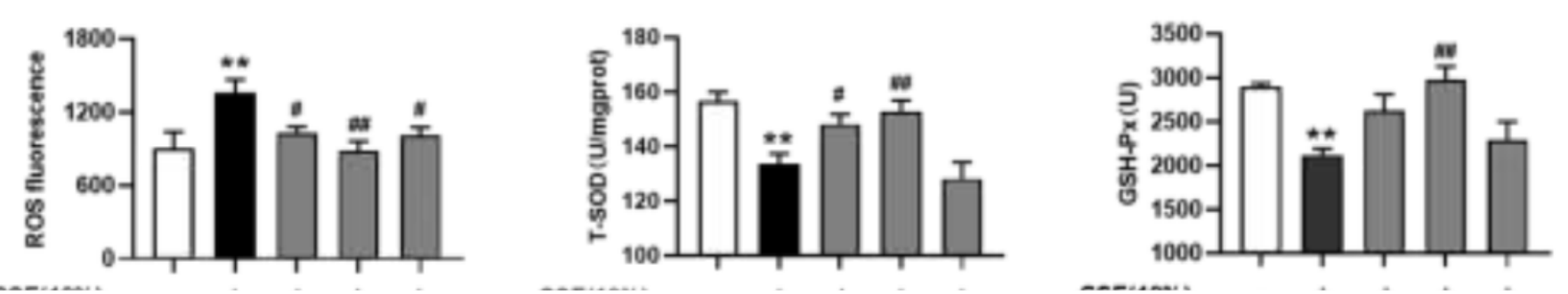

\section{Figure 6}

Effects of ECC-BYF $\mathbb{X}$ on mitophagy and oxidative stress. (A) ROS levels and the activity of T-SOD and GSH-PX; (B) The protein expression of Nrf2 and HO-1. The data are expressed as the mean \pm SE, $(n=3-7)$. ${ }^{* *} P<0.01$ vs. the normal group, ${ }^{*} P<0.05$ vs. the normal group; ${ }^{\#} P<0.01$ vs. the model group, ${ }^{\#} P<0.05$ vs. the model group.

\section{Figure 7}

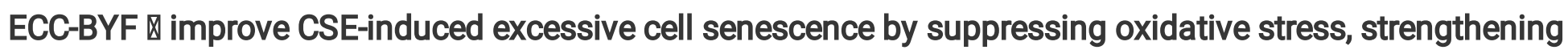
mitophagy. (A) The fluorescence intensity of ROS after intervention with Luteolin; (B) The protein expressions of Nrf2 and HO-1 after intervention with Luteolin; (C) The protein expressions of PINK1, PARK2, Mfn2, Drp1 after intervention with Luteolin; (D) The protein expressions of p16 and p21 after intervention with Luteolin. The data are expressed as the mean $\pm \mathrm{SE},(\mathrm{n}=3) .{ }^{* \star} P<0.01$ vs. the normal group, ${ }^{*} P<0.05$ vs. the normal group; ${ }^{\#} P<0.01$ vs. the model group, ${ }^{\#} P<0.05$ vs. the model group; $\triangle \triangle P<0.01$ vs. the ECC-BYF $\otimes$ treatment group, $\triangle P<0.05$ vs. the ECC-BYF $\otimes$ treatment group. 